SFB

Asymmetry and performance

823

metrics for equity returns

Roger J. Bowden, Peter N. Posch,

Daniel Ullmann

Nr. 26/2016
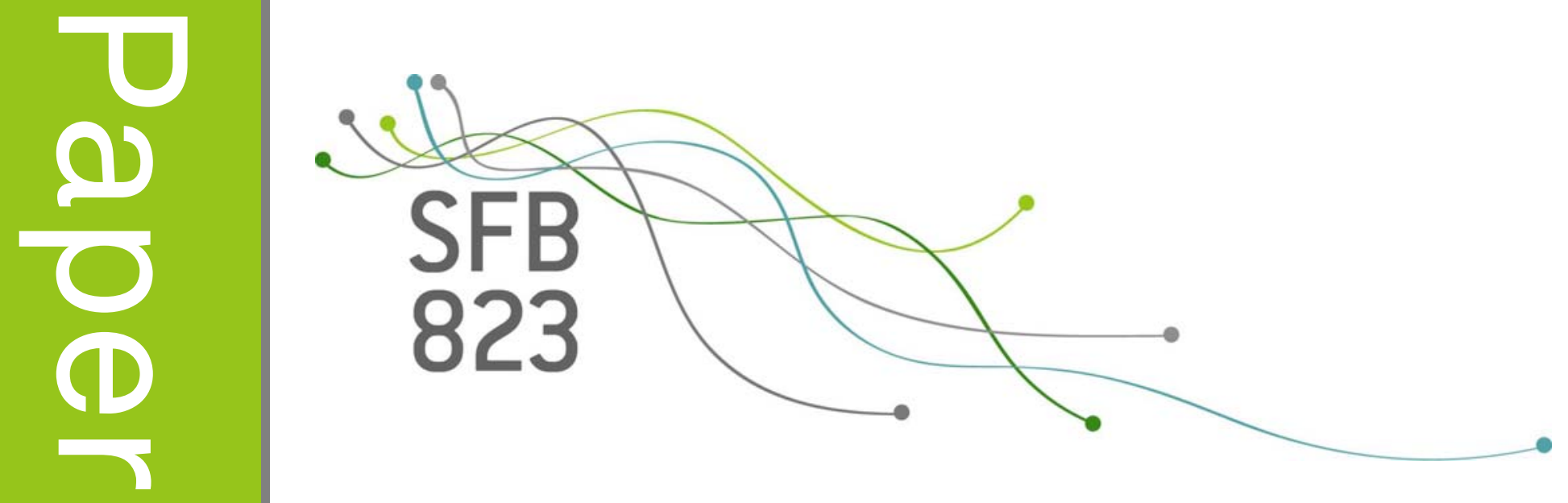



\title{
Asymmetry and performance metrics for equity returns
}

\author{
Roger J. Bowden*, Peter N. Posch ${ }^{\dagger}$, Daniel Ullmann
}

2. Juni 2016

\begin{abstract}
An assumption of symmetric asset returns, together with globally risk averse utility functions, is unappealing for fund managers and other activist investors, whose preferences switch between risk aversion on the downside and risk seeking on the upside. A performance return criterion is originated that is more consistent with the implicit Friedman-Savage utility ordering. Adapted from recent developments in the income distribution literature, the proposed metric weights the lower versus upper conditional expected returns, while a dual spread or dispersion metric also exists. The resulting performance metric is easy to compute. A point of departure is the conventional Sharpe performance ratio, with the empirical comparisons extending to a range of existing performance criteria. In contrast, the proposed $W$-metric results in different and more embracing performance rankings.
\end{abstract}

Keywords: Asymmetry, equity returns, Friedman-Savage utility, fund performance measures, Sharpe ratio, $W$-metric

JEL Classification: C1, G10, G11

Acknowledgement: Financial support by the Collaborative Research Center "Statistical Modeling of Nonlinear Dynamic Processes" (SFB 823) of the German Research Foundation (DFG) is gratefully acknowledged.

\footnotetext{
*Kiwicap Research ltd, Wellington, New Zealand, ph+64 44724094, fax 4983, Email: roger.bowden@kiwicap.co.nz

${ }^{\dagger}$ Technical University of Dortmund, Faculty of Business, Economics and Social Science, 44227 Dortmund, Germany, E-Mail: peter.posch@udo.edu

‡Technical University of Dortmund, Faculty of Business, Economics and Social Science, 44227 Dortmund, Germany, E-Mail: daniel.ullmann@udo.edu, Corresponding author
} 


\section{Introduction}

Performance metrics for equities or managed funds are established tools of the finance industry, objects of media reporting and investor assessment. The metrics in question are usually comparative in nature against some benchmark. For example, the Sharpe (1966) index compares the mean of the security return against the risk free rate, effectively the expected return on a portfolio long in the security return, short in the risk free rate. Likewise, Jensen's alpha looks for positive discrepancies relative to the beta adjusted market return, provided one is confident that a CAPM style equilibrium actually exists (Jensen, 1968). Even short of this, however, there are some hidden assumptions in the use of standard metrics. In the case of the Sharpe index, it is tacitly assumed that the distribution of equity returns is symmetric. But this is not necessarily true, even in relatively normal times (Chunhachinda et al., 1997; Fama, 1965). If returns are asymmetric over any given interval, then extraordinary exposures to loss, or else opportunities for gain, can arise. Kraus and Litzenberger (1976) proposed skewness as a second factor in the traditional Sharpe-Lintner CAPM (Lintner, 1965; Sharpe, 1966). Ang and Chua (1979) use this modification in order to define an excess return performance measure. For other approaches incorporating skewness, see Eling and Schuhmacher (2007); Farinelli et al. (2008).

Any such diagnostic metric should be contextual in nature, with reference to the investor's gain or loss, in a way that the textbook third order moment for asymmetry cannot. And there is room for exploration as to just what should be assumed, even tacitly, about the underlying utility function. A case could be made that the standard concave (riskaverse) utility function is not a comprehensive representation for investor motivation. More appropriate might be a utility function that is concave on the downside and convex on the upside (Friedman and Savage, 1948; Kahneman and Tversky, 1979). Thus a fund manager would be averse to losses, the more so with the prospect of employment termination if losses are heavy. On the other hand, the same manager will be motivated by a more generous performance bonus for progressive gains on the upside.

The purpose of the present paper is to introduce a performance function that addresses such objectives. The methodology is an adaptation of a metric for economic disadvantage recently introduced in the context of income distribution by Bowden (2016b). The adaptation to asset returns adjusts for differing motivational perspectives; too many higher incomes are considered bad for social welfare, but high returns are good for investors! A further normalisation adjustment allows a ready comparison with the corresponding Sharpe metric. The two are the same wherever the return distribution is symmetric; but they differ where this is not the case. Relative to the proposed w-metric, the Sharpe metric understates the investor gains when returns are negatively skewed, i.e. where more distributional weight accrues to higher values, with the reverse effect where the distribution is positively skewed. An empirical analysis shows that the same understatement occurs with a range of other performance metrics that have been proposed, both through 
time and across an extended range of equities. In this sense the proposed w-metric adds a more embracing dimension to fund performance comparisons.

The scheme of the paper is as follows. Section 2 describes the decision theory and distribution properties behind the proposed performance measure, encompassing the double smoothing property of the lower and upper conditional expectations. Section 3 defines and motivates the new performance measure, with the classic Sharpe ratio as a point of departure. The discussion extends to other measures that have been proposed in the literature. An extended empirical illustration in section 4 compares and contrasts the various metrics in detail, in the context of spanning fro beneficial asymmetry. Section 5 contains come concluding remarks.

\section{Metrics and investor utility: the conceptual framework}

The development that follows is general in nature. The basic framework of an investor profit or loss function based on upper and lower conditional expectations is exposited on a general level, followed by the connection with Friedman-Savage decision theory. Taking expected values results in the proposed metric for returns asymmetry, with an accompanying dual metric for spread or dispersion.

\subsection{The win function}

The proposed metric can be cast in terms of the expected value of a profit or loss function. Let $x$ represent any outcome (e.g. income, return or any relevant measurable outcome). The associated random variable $X$ has distribution function $F(x)$ with density $f(x)$. For expositional purposes assume $-\infty<x<\infty$ but the treatment can adapt to the half line $0<x<\infty$ or any compact range. Since our proposed measure is based on the first conditional moment, we shall always assume the existence of the first moment of the associated random variable $X$. For most practical purposes, this is a weak assumption but it does rule out exceptionally long tailed distributions e.g. the Levy. But it suffices for existence of the proposed skewness parameter for empirical density functions, as well as for random variables for which higher moments do not exist (e.g. a t-distribution with $d f=2)$.

For a given value $X=x$, define the progressive conditional expected values as

$$
\begin{aligned}
& \mu_{l}(x)=\mathbb{E}_{F}[X \mid X \leq x]=\frac{1}{F(x)} \int_{-\infty}^{x} X \mathrm{~d} F(X) \quad \text { (left conditional expectation value) } \\
& \mu_{r}(x)=\mathbb{E}[X \mid X>x]=\frac{1}{1-F(x)} \int_{x}^{\infty} X \mathrm{~d} F(X) \quad \text { (right conditional expectation value) }
\end{aligned}
$$

As $x \rightarrow \infty, \mu_{l}(x) \rightarrow \mu$ and $\mu_{r}(x) \rightarrow x \rightarrow \infty$, while for $x \rightarrow-\infty, \mu_{l}(x) \rightarrow x \rightarrow-\infty$ and $\mu_{r}(x) \rightarrow \mu$. Figure 1 illustrates with a logistic distribution, which has fat tails relative to the normal distribution; in this case the asymptotic convergence is very slow. Expression 
(1) as the conditional lower moment has found application in fund performance (see section 3.2). In contrast, the present application utilises both upper and lower conditional moments, with a further stage of consolidation into a single valued metric. The function $v(x)=\left(\mu_{r}(x)-x\right)-\left(x-\mu_{l}(x)\right)$ is referred to in the recent income distribution literature as the net economic disadvantage function. In this context, suppose my income is $x$. I feel disadvantaged to the extent of the average income above mine $\left(\mu_{r}(x)-x\right)$, but net of those below me $\left(x-\mu_{l}(x)\right)$.

In an investment context, where $x$ refers to returns, things have to be reversed. On any given day suppose the return is $x$. Now I win to the extent that this exceeds the average return below (other days are worse), and I lose to the extent that it falls short of the average above (other days are better). This indicates that a more appropriate welfare function in the finance context is the negative of the net economic disadvantage function, i.e.

$$
w(x)=-v(x)=\left(x-\mu_{l}(x)\right)-\left(\mu_{r}(x)-x\right),
$$

the 'win' function. The function $w(x)$ is concave below (convex above) a break-even value on the return axis. As $x \rightarrow-\infty$ it become asymptotic from above to the 45 line, and from below as $x \rightarrow \infty$. The zero point, where it crosses the $x$-axis, is distribution specific (see figure 1). An indication of its position can be inferred from the relationship

$$
w\left(x_{m}\right)=2\left(x_{m}-\mu\right)
$$

where $\mu=\mathbb{E}(x)$ is the expected value and $x_{m}$ is the median. Thus if $\mu<x_{m}$, which will usually be the case with negative skewness, then has its zero to the left of the median, conversely if . For a symmetric distribution crosses at the common mean and median, while the function itself is anti-symmetric about the x-axis. The logistic is an example as in figure 1.

\section{$<$ insert figure 1 about here $>$}

Figure 1: The function $w(x)$ illustrated with a logistic distribution. For clarity, the values have been scaled.

As earlier remarked, lower concavity versus upper convexity is a universal property (Bowden, 2016a,b). This motivates the description of the function $w(x)$ as a FriedmanSavage utility function. However, there are some qualifications as to scope. The classical utility function is distribution invariant, but in the present context the function $w(x)$ depends on the conditional expectation functions $\mu_{l}(x), \mu_{r}(x)$ and therefore upon the distribution $F(x)$. In this sense it could be interpreted as supplementary guide to decision making. Faced with a given returns distribution, the investor could use it to decide whether or not the implied $w(x)$ utility function is consistent with his or her own preferences as between profits and losses. 


\subsection{The expected value}

The expected value of the function $w(x)$ results in a single valued metric for investor profit or loss:

$$
w=\mathbb{E}[w(x)]=\int_{-\infty}^{\infty} w(x) \mathrm{d} F(x) .
$$

One could imagine an investor selecting a day at random and deriving a return $x$ on that day. Over any such random choice of day, expression (5) gives the expected gain or loss. The integral in (5) represent a double smoothing process. The first smoothing level (expressions 1,2) is to obtain the conditional expected value to the left and right of any chosen value $x$. The second is to smooth the results over all values of $x$.

However, the two stages can be condensed into a simple one stage expectation by means of a change of measure. The left and right entropic shifts of $F(x)$ are distributions defined by

$$
F_{L}(y)=F(y)\left(1+\xi_{L}(y)\right) ; \quad 1-F_{R}(y)=(1-F(y))\left(1+\xi_{L}(y)\right)
$$

where the shift factors ( technically Radon-Nikodym derivatives) are given by

$$
\xi_{L}(y)=-\ln F(y) ; \quad \xi_{R}(y)=-\ln (1-F(y))
$$

Properties of the left and right entropic shifts can be found in Bowden $(2012,2016 \mathrm{~b})$. In such terms,

$$
\mathbb{E}\left(\mu_{l}(x)\right)=\int_{-\infty}^{\infty} \mu_{l}(x) \mathrm{d} F(x)=\int_{-\infty}^{\infty} x \mathrm{~d} F_{L}(x)=\mu_{L}
$$

the simple mean of the left entropic shift. Similarly,

$$
\mathbb{E}\left(\mu_{r}(x)\right)=\int_{-\infty}^{\infty} \mu_{r}(x) \mathrm{d} F(x)=\int_{-\infty}^{\infty} x \mathrm{~d} F_{R}(x)=\mu_{R},
$$

the simple means of the shifted distributions defined by expressions $(6,7)$. Expressions $(8,9)$ embody the double smoothing property. The first layer is the progressive smoothing up to or beyond any given point $x$. The second layer smoothes the result over all such points, using the density as a weighting kernel.

The expected value of the net win function is therefore

$$
w=2\left(\mu-\frac{1}{2}\left(\mu_{L}+\mu_{r}\right)\right)
$$

An implicit asymmetry test is apparent in the form of (10). The entropic shifts $F_{L}, F_{R}$ represent bodily displacements to the left and right of the natural distribution $F(x)$. If more entropic (uncertainty) mass is contained to the left, then the displacement difference is greater than to the right and the average of the two means will be less than the 
original. Thus a distribution $F(x)$ that is naturally negatively skewed will have $w>0$, while positive skewness will lead to $w<0$. For any symmetric distribution, $w=0$. Outcomes of this kind correspond to classical metrics such as the Pearson third order moment. Unlike the standard cubic, however, there is now an explicit contextual reference, as to how investors might view things in welfare or utility terms.

\subsection{An associated dispersion metric}

A simple change of sign in expression (3) provides a penalty function for spread or dispersion (Bowden, 2016b). This is defined as

$$
d(x)=\left(x-\mu_{l}(x)\right)+\left(\mu_{r}(x)-x\right)=\mu_{r}(x)-\mu_{l}(x) .
$$

It follows from their respective definitions that $d(x)>w(x)$.

Taking the expected value gives a metric for dispersion:

$$
d=\mathbb{E}(d(x))=\mu_{R}-\mu_{L}>0
$$

The underlying dispersion concept is based on the total partition entropy of the distribution. The partition entropy function $h(x)$ at any chosen point represents the uncertainty as to whether $X>x$ or $X<x$. The partition entropy function has itself given rise to a number of applications in areas such as a smoothing kernels or scaling algorithms. Integrating $h(x)$ over $x$ gives the metric $d$, which can be interpreted as the total entropic spread of the distribution. There is also a covariance connection with asymmetry, as measured by the metric $w$. A distribution is negatively skewed if the dispersion function $d(x)$ is greater in the region where $F(x)<0.5$, so the covariance between $d(x)$ and $F(x)$ is negative.

\section{Performance metrics}

The foregoing definitions and interpretations are employed in the present section to define and motivate the proposed performance metric as an alternative to the standard Sharpe measure. Section 3.2 extends the comparison to other existing performance metrics.

\subsection{The $W$-metric}

The basic Sharpe performance index serves as a starting point for a more general discussion. As we are focusing on investment performance, the general outcome $x$ is replaced by the context specific return $r$. The standard Sharpe metric is written as

$$
S(r)=\frac{\mathbb{E}(r)-r_{f}}{\sigma(r)}
$$


where $\mathbb{E}(r)$ is the expected return, $\sigma$ its standard deviation and $r_{f}$ is a risk free rate. Defining $\tilde{r}=r-r_{f}$ as the excess return, with distribution function $\tilde{F}$, the Sharpe ratio becomes

$$
S(\tilde{r})=\frac{\mathbb{E}_{\tilde{F}}(\tilde{r})}{\tilde{\sigma}},
$$

where $\tilde{\sigma}$ denotes the standard deviation of excess returns.

The proposed $W$-metric is an alternative to the Sharpe ratio that explicitly encompasses the positive or negative benefits of asymmetry. It is defined as

$$
W(r)=\frac{w(\tilde{r})+\tilde{r}_{m}}{d(\tilde{r})}:=\frac{\tilde{w}+\tilde{r}_{m}}{\tilde{d}}
$$

where $\tilde{r}_{m}$ is the median of the excess return distribution and $w, d$ are defined according to expressions (10) and (12). The denominator $d$ of the $W$-metric is adapted to the entropic interpretation of the asymmetry metric $w$ in the numerator. For standardisable distributions such as the Normal, the logistic or the Gumbel, the metrics $d$ and the standard deviation $\sigma$ are proportional via the scale parameter. In such cases there is little effective difference between using either $d$ or $\sigma$ for the denominator.

The numerator of the metric (15) splits into two terms: $\tilde{w}$ captures the asymmetry of the excess returns distribution while $\tilde{r}_{m}$ adds the median excess return. Rewriting the numerator of $W$ results in:

$$
\tilde{w}+\tilde{r}_{m}=\left(\mathbb{E}(r)-r_{f}\right)+\left[\tilde{w}+r_{m}-\mathbb{E}(r)\right]
$$

Thus the $W$-metric and the Sharpe ratio's numerator differ in the term $\left[\tilde{w}+r_{m}-\mathbb{E}(r)\right]$. Consider the following cases:

(a) The distribution of excess returns $\tilde{r}$ is symmetric. In this case $\tilde{w}=0, r_{m}=\mathbb{E}(r)$, and the numerators of the $W$-metric and Sharpe are identical.

(b) The distribution of excess returns is positively skewed. In this case $\tilde{w}<0$, and it is likely that $r_{m}-\mathbb{E}(r)<0$. Together this means that $W<S$.

(c) The distribution of excess returns is negatively skewed. In this case, $\tilde{w}>0$, and it is likely that $r_{m}-\mathbb{E}(r)>0$. Together this means that $W>S$.

Cases (b) and (c) reflect the implied Friedman-Savage investor utility basis: investors would like a positively skewed distribution; increasing marginal utility in the higher zone. But they might back away from a negatively skewed one; too much weight in the low zone, the area of more negative marginal utility.

The difference between $W$ and $S$ is generated by the implicit underlying utility functions, and the way that these are responsive to the distribution of returns. The Sharpe metric $S$ tacitly assumes a linear utility function, while the $W$ metric is more responsive to the mixed concave-convex Friedman-Savage type utility function. 
Adaptations of $W$ can be devised for other benchmarks. Instead of using the risk free rate, the comparator could be the market return $R$, so $\tilde{r}=r-R$ is a compound return, long in the subject security and short in the market. Alternatively if a CAPM is thought to exist, a generalisation of Jensen's alpha can be defined with the comparator return as the market, scaled by the security's beta.

\subsection{Other performance metrics}

The empirical work of section 4 encompasses a range of alternative performance metrics that have been proposed in the literature. Two of these, namely the Treynor ratio $\left(\mathbb{E}(r)-r_{f}\right) / \beta$ and Jensen's alpha $\left(\mathbb{E}(r)-r_{f}\right)-\beta\left(r_{m}-r_{f}\right)$ require a more or less explicit CAPM reference.

Of those that do not, the Sortino and Kappa 3 ratios (Kaplan and Knowles, 2004; Sortino et al., 1999) have a point of connection in that in drawing on lower and upper conditional partial moments. Given a threshold return $z$, the lower partial moments of order $n$ with the corresponding threshold return $z$ are defined (c.f. Harlow 1991) as

$$
\operatorname{LPM}_{n}(z)=\frac{1}{F(z)} \int_{-\infty}^{z}(z-x)^{n} \mathrm{~d} F(x)
$$

For the Sortino and Kappa 3 ratios:

$$
\operatorname{Sortino}(z)=\frac{\mathbb{E}(r)-z}{\sqrt[2]{\text { PM }_{2}(z)}} \quad \text { resp. } \quad K_{3}(z)=\frac{\mathbb{E}(r)-z}{\sqrt[3]{L P M_{3}(z)}} .
$$

The upside potential ratio (UPR) employs the higher partial moment of order one:

$$
\operatorname{UPR}(z)=\frac{\frac{1}{F(z)} \int_{z}^{\infty}(z-x) \mathrm{d} F(x)}{\sqrt[2]{\operatorname{LPM}_{2}(z)}}
$$

All versions require the user to specify a benchmark value $z$, so these are not global metrics.

A further comparator is the omega ratio of Keating and Shadwick (2002). In the present notation this can be expressed as

$$
\Omega(z)=\frac{\int_{z}^{\infty}(1-F(x)) \mathrm{d} F(x)}{\int_{-\infty}^{z} F(x) \mathrm{d} F(x)}:=\frac{\Phi_{r}(z)}{\Phi_{l}(z)}
$$

The function $\Phi_{l}(z)$ will be familiar from stochastic dominance theory: distribution A is second order stochastic dominant over B if $\Phi_{l, A}(z) \leq \Phi_{l, B}(z)$ for all $z$. Keating and Shadwick propose a less stringent version: the investor sets a threshold value of $z$, below which outcomes are regarded as losses and above as gains and compares the ratio (20) relative to that threshold.

There is no direct connection between the Omega ratio and the present $W$-metric, though the functions $w(x), d(x)$ utilised in the latter help to determine the shape of $\Omega(x)$ via 
the differential equation

$$
\frac{\mathrm{d} \log \Omega(x)}{\mathrm{d} x}=-4 \frac{d(x)}{d^{2}(x)-w^{2}(x)}
$$

The omega ratio function is evidently a compounding of both spread and asymmetry, with the leading effect of order $d^{-1}(x)$. If the dispersion is high, a unit step to the right on the return axis does not increase the probability for losses that much, compared to a very dense distribution, where a unit step covers more probability mass.

A final group of comparators utilises the notion of maximum drawdown $(M D)$ (Magdon-Ismail et al., 2003). A drawdown is the maximal loss in a given time span with a designated end date and a variable start date within the given time span. The maximum drawdown denotes the maximum of the drawdowns within the given time span. Three variants utilised in the empirical comparisons of section 4 are as follows:

$$
\begin{aligned}
\text { Calmar ratio }(r) & =\frac{\mathbb{E}(r)-r_{f}}{-M D} \\
\text { Sterling ratio }(r) & =\frac{\mathbb{E}(r)-r_{f}}{\mathbb{E}(-M D)} \\
\text { Burke ratio }(r) & =\frac{\mathbb{E}(r)-r_{f}}{\sqrt{\sum-M D}}
\end{aligned}
$$

\section{Empirical application}

The empirical work that follows proceeds in two phases. Section 4.1 looks at the relative performance of the $W$-metric with the conventional Sharpe ratio as the point of departure. For this more limited comparison, historical returns on just a single equity are used. Section 4.2 is an extended set of comparisons with the range of alternative metrics as in section 3.2, applied to the complete regimen of equities in the S\&P500.

\subsection{Comparison with the Sharpe ratio}

To illustrate the new measure, we use the daily log returns of Ford Motors Company together with the S\&P500 index as a market proxy. The 10 year US treasury yield is employed as a proxy for the risk free rate from 1990 to 2015. Table 1 gives descriptive statistics. For all the different log return distributions, any evident skewness has the sign expected in the $w$ statistic of equation (5)

$<$ insert table 1 about here $>$

Table 1: Descriptive statistics and the corresponding $w$ statistic for the daily returns distribution

Turning to explicit performance indicators, the Sharpe ratios and the $W$ metrics are compared for two benchmarks: the risk free rate and the market return. The results are 
shown in figure 2. The Sharpe and $W$ measures are calculated using for daily returns over each different year. The upper chart (a) utilises the risk free rate as benchmark as in the classic Sharpe ratio. The dashed bar corresponds to $W$ in equation (15) and the solid bar to the Sharpe ratio. The two measures have a correlation coefficient of $\rho_{S, W}=0.496$ at a significance level of $\alpha=1 \%$. However, there are several occurrences (1990, 1991 and 2007) where not only the absolute values between the Sharpe and the $W$-measure differ, but also the signs.

$<$ insert figure 2 about here $>$

Figure 2: Comparison for the $W$-metric and the Sharpe ratio and (a) the risk-free rate and (b) the market return as comparator benchmarks.

As discussed in section 3, one can look for two scenarios where $W$ is potentially unequal to $S$. In the sample there are 13 years with a positive skewness, of which 12 years confirm the prediction $S>W$. Only in the one year 2005 does the Sharpe ratio exceed $W$. Turning to the likelihood of having $r_{m}-\mathbb{E}(r)<0$, there is an empirical 'hit ratio' of 10 out of 13 cases $(79.6 \%)$. In the case of negative skewness, the $W>S$ prediction is confirmed in 9 out of 13 years. The empirical likelihood of having $r_{m}-\mathbb{E}(r)>0$ with $\tilde{w}>0$ is at $46.2 \%$. The lower chart (b) of figure 2 utilises the market return as a comparator. To clarify the difference relative to the risk free rate, the resulting metrics are denoted $S^{*}$ and $W^{*}$. The dashed bar corresponds to $W^{*}$ and the solid bar to the Sharpe analogue ratio $S^{*}$. In 9 out of 26 observations the two have different signs along with an insignificant correlation coefficient of $\rho_{S^{*}, W^{*}}=0.243$. For the positive skewness we find $S^{*}>W^{*}$ in 11 out of 14 years. All 14 years, have $\tilde{w}<0$ while in 11 years, $r_{m}-\mathbb{E}(r)<0$ i.e. an empirical concordance likelihood of $78.6 \%$. In the 12 years with a negative skewness, 11 years have $W^{*}>S^{*}$ and the same 11 years show $r_{m}-\mathbb{E}(r)<0$. Only two years show $r_{m}-\mathbb{E}(r)>0$; thus an empirical correspondence of only $18.2 \%$.

As previously discussed, the standard deviation can be used as denominator instead of the dispersion metric $d$, with the performance indicator as $W_{S}=\frac{\tilde{w}+\tilde{r}_{m}}{\tilde{\sigma}}$. The analysis is then repeated using $W_{S}$ instead of $W$. The result is shown in figure 3 . In all cases, the sign is preserved, i.e. $\operatorname{sgn}(W)=\operatorname{sgn}\left(W^{*}\right)$, but the absolute value of $W_{S}$ is always larger. Using the standard deviation therefore leads to a higher value of the metric. For the upper chart, there are three cases where the alternative definitions lead to different conclusions as to the size of the metric. In 2005, 2008 and 2010 we find $|W|<S<\left|W_{S}\right|$ . For the lower part of figure 3 the years 1994, 2005 and 2008 show a similar pattern.

$<$ insert figure 3 about here $>$

Figure 3: Comparison for the $W$-metric and the Sharpe ratio with STD as denominator, and (a) the risk-free rate and (b) the market return as comparator benchmarks. 


\subsection{Other literature metrics: a more extended comparison}

Turning to the more extended set of measures described in section 3, performance comparisons utilise daily returns on the complete constituent companies of the S\&P500, listed as of $11^{\text {th }}$ November 2015, adjusting where necessary for missing data. The final data spans 20 years of daily data on 366 stocks, in total 6222 distributions each spanning one year of daily returns. Descriptive statistics are shown in table 2.

$<$ insert table 2 about here $>$

Table 2 Descriptive statistics for the 6222 daily return distributions

Shown are the mean, median, standard deviation, minimum \& maximum, skewness and kurtosis of the annual percentage equivalent daily returns. Using the Jarque-BeraTest, $82.5 \%(87.2 \%)$ of the distributions are indicated as non-normally distributed, at a significance level of $1 \%(5 \%)$.

As earlier noted, the performance comparisons studies encompass a more extended set of measures that have been proposed in the literature. These are chosen as the Sortino ratio, Kappa 3, the UPR, the Calmar ratio, Sterling ratio, Burke ratio, Jensen's alpha and the Treynor ratio to the Sharpe ratio and the Omega ratio.

For the Omega ratio, the Sortino ratio, the Kappa 3 and the UPR, we use the return distribution as the P\&L distribution, e.g. we set the threshold to a value of $z=0$. For Jensen's alpha and the Treynor ratio, the CAPM beta coefficient is estimated for each year using the corresponding stock returns and the S\&P500 returns as proxy for the market return. Technically this implies a forward looking beta coefficient, but the results do not change when using the lagged return distributions.

Comparative results are shown in table 3. Reported is the lower triangle rank correlation matrix. Considering the first row of averaged rank correlation enables comparison with the results of Eling and Schuhmacher (2007). In general conformity with their findings, we find very high and significant correlations with most metrics. For Jensen's alpha, there is a lower rank correlation of 0.68 on average, compared to 0.94 in their study. For the Treynor ratio, we find a higher rank correlation of 0.82 on average, compared to their 0.32 .

Introducing the $W$ and $W^{*}$ measures as defined in section 3, gives rise to the last three rows in table 2 . The rank correlations are now significantly lower than for the other measures. For the $W$ measure, there is an average rank correlation of 0.25 and for the $W^{*}$ measure an average rank correlation of 0.12 . The proposed 'win' metrics therefore differ significantly in the ranking form the existing measures. Since the Sharpe ratio is known for a skewness bias, the rankings of the other measures may likewise influenced by the skewness of the distribution. Once this is explicitly incorporated via the $W$ metrics, things do change. If investors or fund managers prefer negative skewness, as argued in section 2 , then the $W^{*}$ metric provides a better set of rankings across different equity returns. 


\section{Concluding remarks}

Investors naturally seek high returns and low volatility, and there is a case for negative skewness in addition. In order to evaluate return distributions, we propose the ratio of a contextually motivated skewness metric to dispersion. The latter can be either as the dual spread metric or else just the standard deviation. A sensitivity analysis shows that the bias arising from skewness in the Sharpe ratio does not crucially rely on the definition of the denominator. Thus in the Ford Motors illustration, the pattern changes in only in $11 \%$ of the cases.

Particular motivation for the augmented $W$-metric exists in episodes where skewness can arise, as in times of market stress. However even in more normal times, it is useful to be able to separate out the separate contribution of skewness as a potentially important dimension to portfolio analysis. This is especially so where a given stock or ETF portfolio displays habitual tendencies in one direction or the other. The advantage of thinking in Friedman-Savage terms is that it does focus on the welfare consequences of investment decisions in the presence of potential skewness. Once we allow for investor preferences of this kind, the rankings of alternative investments can change quite dramatically. 


\section{A. Figures and Tables}

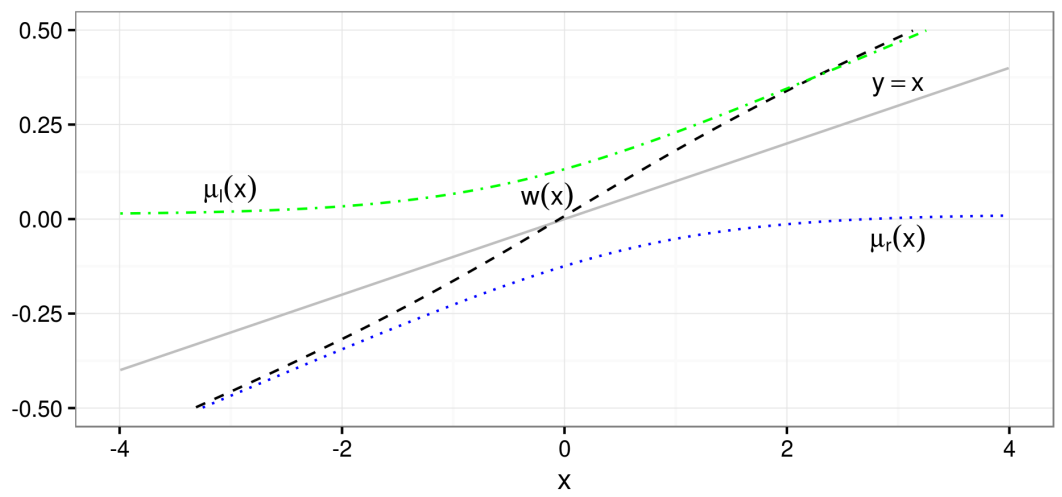

FIG. 1: The function $w(x)$ illustrated with a logistic distribution. For clarity, the values have been scaled. 


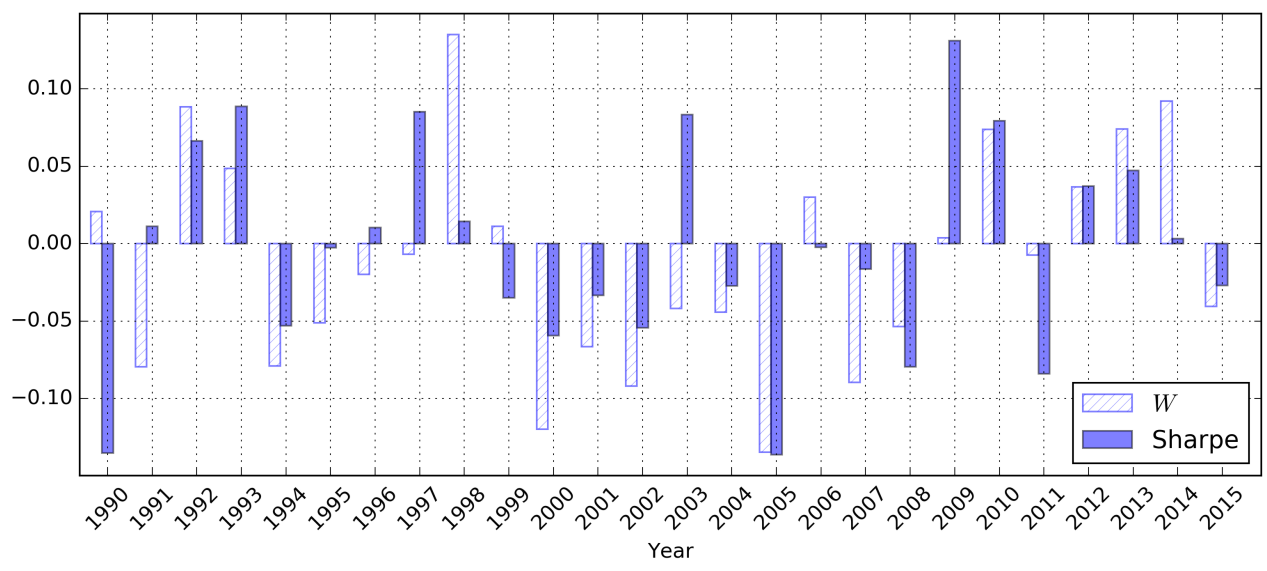

(a) Risk free rate

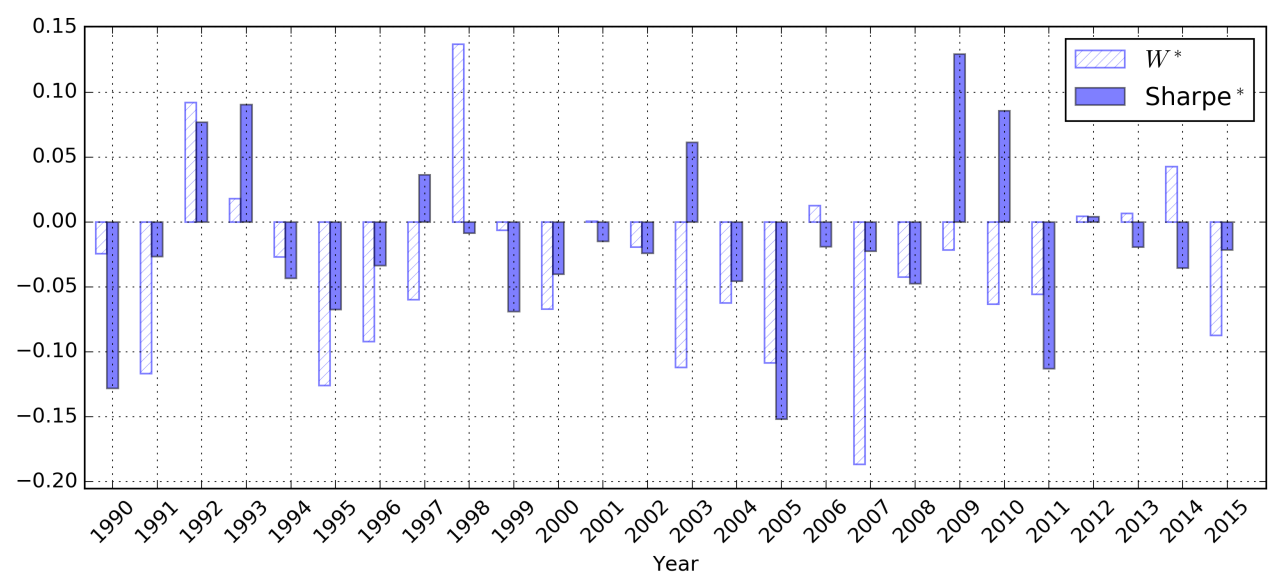

(b) Market return

FIG. 2: Comparison of the $W$-metric and the Sharpe Ratio for (a) the risk-free rate and (b) the market return as comparator benchmarks. 


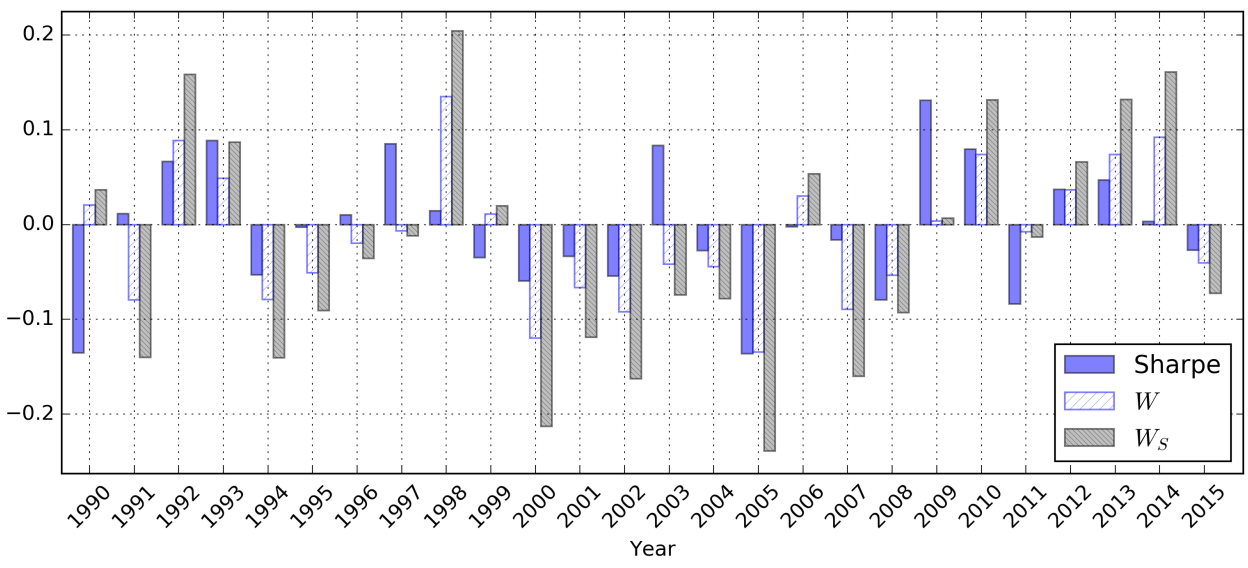

(a) Risk free rate

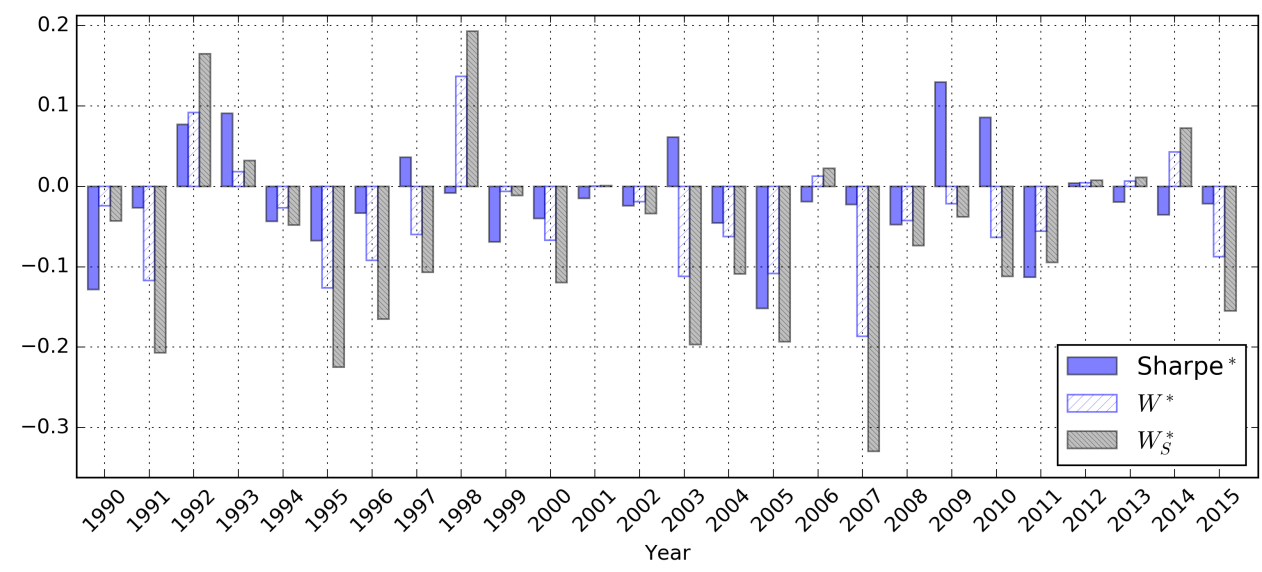

(b) Market return

FIG. 3: The $W$-metric and the Sharpe Ratio: STD as denominator, and (a) the risk-free rate and (b) the market return as benchmarks. 
ТАв. 1: Descriptive statistics and the corresponding w measure for the daily returns distribution. All values are given as \%.

\begin{tabular}{rrrrrrrrrrrrrr}
\hline & 1990 & 1991 & 1992 & 1993 & 1994 & 1995 & 1996 & 1997 & 1998 & 1999 & 2000 & 2001 & 2002 \\
\hline Mean & -0.21 & 0.04 & 0.16 & 0.17 & -0.07 & 0.01 & 0.03 & 0.16 & 0.07 & -0.05 & -0.15 & -0.07 & -0.17 \\
Median & 0.00 & 0.00 & 0.28 & 0.21 & -0.19 & 0.00 & 0.00 & 0 & 0.18 & 0 & -0.35 & -0.28 & -0.37 \\
SD & 1.73 & 2.01 & 2.11 & 1.7 & 1.73 & 1.66 & 1.51 & 1.65 & 3.49 & 2.01 & 2.84 & 2.39 & 3.31 \\
Skewness & -0.4 & 0.85 & -0.21 & 0.16 & 0.07 & 0.43 & 0.16 & 0 & -5.44 & -0.17 & 0.51 & -0.06 & 0.46 \\
Skewness of excess return & -0.87 & 0.77 & -0.11 & 0.13 & -0.11 & 0.4 & 0.23 & 0.18 & -7.11 & -0.08 & 0.27 & -0.29 & 0.14 \\
$w$ & 0.09 & -0.26 & 0.08 & -0.05 & -0.04 & -0.14 & -0.04 & 0 & 0.55 & 0.06 & -0.23 & 0.01 & -0.15 \\
\hline
\end{tabular}

\begin{tabular}{rrrrrrrrrrrrrr}
\hline & 2003 & 2004 & 2005 & 2006 & 2007 & 2008 & 2009 & 2010 & 2011 & 2012 & 2013 & 2014 & 2015 \\
\hline Mean & 0.22 & -0.03 & -0.23 & 0.01 & -0.02 & -0.47 & 0.56 & 0.2 & -0.2 & 0.06 & 0.08 & 0.01 & -0.03 \\
Median & 0.08 & -0.06 & -0.3 & 0 & -0.24 & -0.64 & 0.28 & 0.28 & -0.16 & 0.06 & 0.1 & 0.06 & -0.13 \\
SD & 2.51 & 1.69 & 1.81 & 2.34 & 2.16 & 6.1 & 4.19 & 2.41 & 2.51 & 1.53 & 1.51 & 1.31 & 1.41 \\
Skewness & 0.64 & 0.6 & 0.57 & -0.64 & 0.26 & -0.08 & 0.17 & -0.09 & -0.64 & -0.17 & -0.48 & -1.13 & -0.18 \\
Skewness of excess return & 1.03 & 0.81 & 0.41 & -0.66 & 0.77 & 0.26 & 0.29 & 0.51 & -1.56 & -0.74 & -1.2 & -1.67 & -0.14 \\
$w$ & -0.25 & -0.06 & -0.12 & 0.14 & -0.1 & 0.08 & -0.24 & 0.04 & 0.14 & 0.04 & 0.11 & 0.16 & 0.03 \\
\hline
\end{tabular}

ТАв. 2: Descriptive statistics for the 6222 daily return distributions

\begin{tabular}{rccccc}
\hline & Mean & Median & Standard deviation & Min & Max \\
\hline Mean / \% & 0.07 & 0.07 & 0.14 & -0.72 & 1.52 \\
Standard deviation / \% & 2.25 & 1.97 & 1.2 & 0.61 & 15.79 \\
Skewness & 0.09 & 0.12 & 1.08 & -12.71 & 15.48 \\
Kurtosis & 4.64 & 2.05 & 11.05 & -0.42 & 240.15 \\
\hline
\end{tabular}


ТАВ. 3: Rank correlations for different performance measures

\begin{tabular}{|c|c|c|c|c|c|c|c|c|c|c|c|c|}
\hline & Sharpe ratio & Omega & Sortino & Kappa & Upside & Calmar & Sterling & Burke & Jensen & Treynor & $W$ & $W^{*}$ \\
\hline Omega & $1.00^{* * *}$ & & & & & & & & & & & \\
\hline Sortino Ratio & $1.00^{* * *}$ & $1.00^{* * *}$ & & & & & & & & & & \\
\hline Kappa 3 & $0.99^{* * *}$ & $0.99^{* * *}$ & $1.00^{* * *}$ & & & & & & & & & \\
\hline Upside potential ratio & $0.89^{* * *}$ & $0.88^{* * *}$ & $0.90^{* * *}$ & $0.92^{* * *}$ & & & & & & & & \\
\hline Calmar ratio & $0.98^{* * *}$ & $0.99^{* * *}$ & $0.99^{* * *}$ & $0.98^{* * *}$ & $0.89^{* * *}$ & & & & & & & \\
\hline Sterling ratio & $0.98^{* * *}$ & $0.99^{* * *}$ & $0.99 * * *$ & $0.99^{* * *}$ & $0.89^{* * *}$ & $1.00^{* * *}$ & & & & & & \\
\hline Burke ratio & $0.99^{* * *}$ & $0.99^{* * *}$ & $1.00^{* * *}$ & $0.99^{* * *}$ & $0.89^{* * *}$ & $0.99^{* * *}$ & $0.99^{* * *}$ & & & & & \\
\hline Jensen's alpha & $0.77^{* * *}$ & $0.77^{* * *}$ & $0.77^{* * *}$ & $0.77^{* * *}$ & $0.70^{* * *}$ & $0.75^{* * *}$ & $0.77^{* * *}$ & $0.76^{* * *}$ & & & & \\
\hline Treynor ratio & $0.95^{* * *}$ & $0.95^{* * *}$ & $0.95^{* * *}$ & $0.94^{* * *}$ & $0.84^{* * *}$ & $0.94^{* * *}$ & $0.95^{* * *}$ & $0.95^{* * *}$ & $0.78^{* * *}$ & & & \\
\hline Average rank correlation & 0.85 & 0.85 & 0.86 & 0.86 & 0.78 & 0.85 & 0.85 & 0.86 & 0.68 & 0.82 & & \\
\hline$W$ & $0.29^{* * *}$ & $0.28^{* * *}$ & $0.26^{* * *}$ & $0.25^{* * *}$ & 0 & $0.29^{* * *}$ & $0.28^{* * *}$ & $0.29^{* * *}$ & $0.14^{* * *}$ & $0.26^{* * *}$ & & \\
\hline$W^{*}$ & $0.10^{* * *}$ & $0.09^{* * *}$ & $0.07^{* * *}$ & $0.06^{* * *}$ & $-0.11^{* * *}$ & $0.09^{* * *}$ & $0.09^{* * *}$ & $0.09^{* * *}$ & $0.20^{* * *}$ & $0.09^{* * *}$ & $0.65^{* * *}$ & \\
\hline Average rank correlation & 0.74 & 0.74 & 0.74 & 0.74 & 0.64 & 0.74 & 0.74 & 0.74 & 0.6 & 0.72 & 0.25 & 0.12 \\
\hline
\end{tabular}

Note: ${ }^{* *} p<0.01,{ }^{* *} p<0.05,{ }^{*} p<0.1$. 


\section{Literature}

Ang, J.S. and Chua, J.H., Composite measures for thte evaluation of investment performance. Journal of Financial and Quantitative Analysis, 1979, 14, 361-384.

Bowden, R.J., Information, measure shifts and distribution metrics. Statistics, 2012, 46, 249-262.

Bowden, R.J., Dual spread and asymmetry distribution metrics based in partition entropy. Kiwcap Research Ltd, 2016a, pp. 1-13.

Bowden, R.J., Giving Gini direction: An asymmetry metric for economic disadvantage. Economics Letters, 2016b, 138, 96--99.

Chunhachinda, P., Dandapani, K., Hamid, S. and Prakash, A.J., Portfolio selection and skewness: Evidence from intemational stock markets. Journal of Banking and Finance, 1997, 21, 143-167.

Eling, M. and Schuhmacher, F., Does the choice of performance measure influence the evaluation of hedge funds?. Journal of Banking and Finance, 2007, 31, 2632-2647.

Fama, E.F., Portfolio Analysis in a Stable Paretian Market. Management Science, 1965, 11, 404-419.

Farinelli, S., Ferreira, M., Rossello, D., Thoeny, M. and Tibiletti, L., Beyond Sharpe ratio: Optimal asset allocation using different performance ratios. Journal of Banking and Finance, 2008, 32, 2057-2063.

Friedman, M. and Savage, L.J., The Utility Analysis of Choices Involving Risk. The Journal of Political Economy, 1948, 56, 279-304.

Harlow, W.V., Asset Allocation in a Downside-Risk Framework. Financial Analysts Journal, 1991, 47, 28-40.

Jensen, M.C., The Performance of Mutual Funds in the Period 1945-1964. Journal of Finance, 1968, 23, 389.

Kahneman, D. and Tversky, A., Prospect theory: An analysis of decision under risk. Econometrica: Journal of the Econometric Society, 1979, 47, 263-292.

Kaplan, P. and Knowles, J., Kappa: A Generalized Downside Risk-Adjusted Performance Measure. Journal of Performance Measurement, 2004, 8, 42-54.

Keating, C. and Shadwick, W.F., A Universal Performance Measure. Journal of Performance Measurement, 2002, 6, 59-84.

Kraus, A. and Litzenberger, R., Skewness preference and the valuation of risky assets. Journal of Finance, 1976, 31, 1085-1100. 
Lintner, J., The valuation of risk assets and the selection of risky investments in stock portfolios and capital budgets. The Review of Economics and Statistics, 1965, pp. 13$-37$.

Magdon-Ismail, M., Atiya, A., Pratap, A. and Abu-Mostafa, Y., The maximum drawdown of the Brownian motion. In Proceedings of the IEEE/IAFE Conference on Computational Intelligence for Financial Engineering, Proceedings (CIFEr), Vol. 2003-Janua, pp. 243247, 2003, Institute of Electrical and Electronics Engineers Inc.

Sharpe, W.F., Mutual Fund Performance. The Journal of Business, 1966, 39, 119-138.

Sortino, F.A., van der Meer, R. and Plantinga, A., The Dutch Triangle. The Journal of Portfolio Management, 1999, 26, 50-57. 


